Techniques \& Culture

\title{
Produire pour offrir
}

L'offrande par destination chez les Celtes

\section{Élisabeth Goussard}

\section{CpenEdition}

Journals

Édition électronique

URL : https://journals.openedition.org/tc/9869

DOI : $10.4000 /$ tc. 9869

ISSN : 1952-420X

Éditeur

Éditions de l'EHESS

\section{Édition imprimée}

Date de publication : 30 octobre 2018

Pagination : 200-213

ISBN : 2-7132-2751-6

ISSN : 0248-6016

Référence électronique

Élisabeth Goussard, «Produire pour offrir », Techniques \& Culture [En ligne], 70 | 2018, mis en ligne le 06 décembre 2020, consulté le 29 septembre 2022. URL : http://journals.openedition.org/tc/9869 ; DOI : https://doi.org/10.4000/tc.9869 


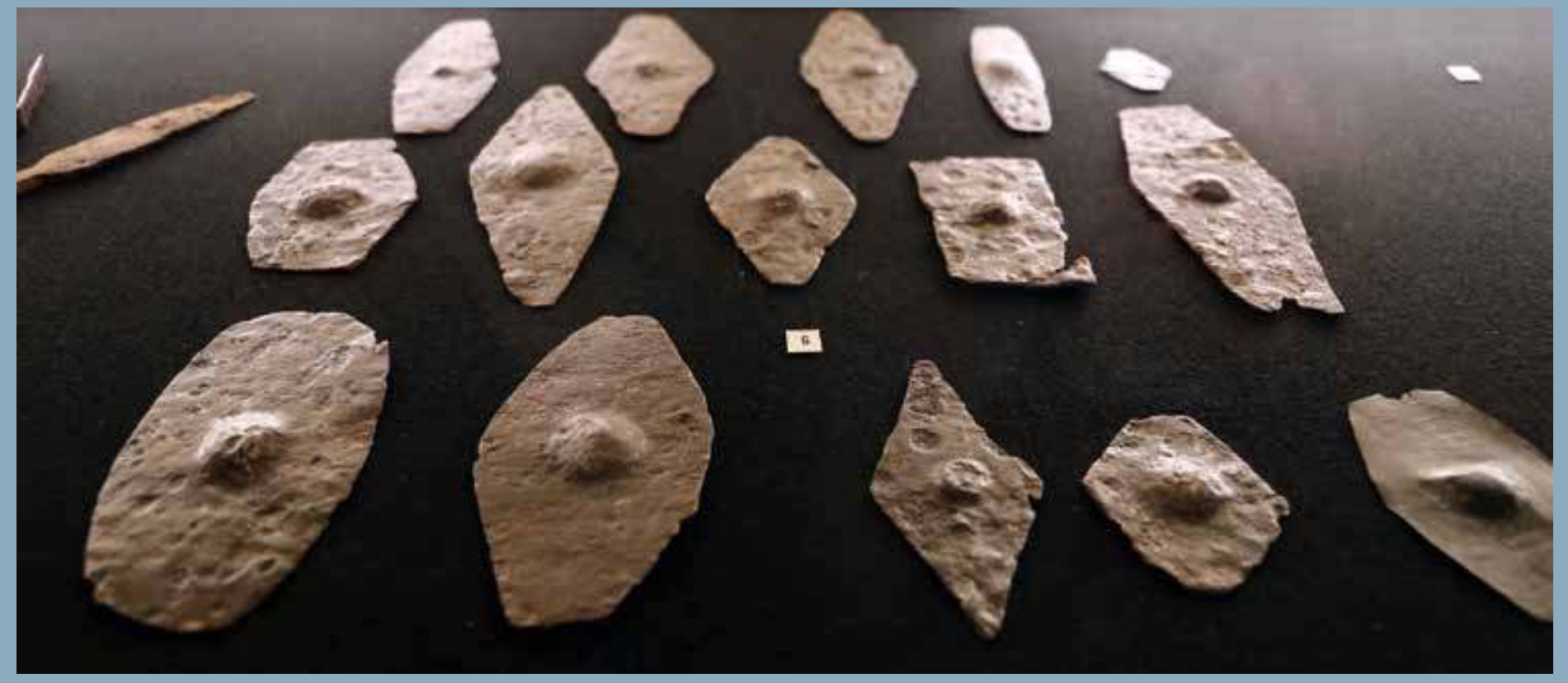




\section{Produire pour offrir L'offrande par destination chez les Celtes}

Les miracles ne font pas que des heureux. Le Liber Prior miraculorum (BHL 4863), au xi siècle, décrit l'irritation d'un garde face aux trop nombreuses libérations surnaturelles provoquées par saint Léonard, dont la seule invocation pouvait faire fondre les entraves. Par conséquent, le geôlier exaspéré dit envisager de creuser une fosse d'une grande profondeur, à l'intérieur de sa tour, et d'y ensevelir son détenu.

Ces libérations miraculeuses ont en revanche fait la fortune de l'abbaye de Noblat, où se trouve le tombeau du saint. Les anciens prisonniers venaient y apporter les chaînes dont ils avaient été délivrés. Certains, comme Bohémond de Tarente, un des meneurs de la première croisade, allaient même jusqu'à offrir des chaînes en métaux précieux, en or et en argent, confectionnées à cet effet (Cheirézy 1995: 429-431).

Les attitudes de ces anciens détenus mettent en évidence l'existence de deux types d'offrandes. Les premières sont des offrandes dites par transformation, c'est-à-dire des objets retirés du monde profane, détournés de leur fonction première, pour être réutilisés comme offrandes. Les anciens fers des prisonniers en sont des exemples. Ils se différencient donc des offrandes par nature ou par destination (Morel 1992, Bataille 2011 : 657), qui ne sont produites que dans le but d'être offertes. Il s'agit ici des chaînes de Bohémond.

De nos jours, les offrandes par destination sont majoritaires dans le paysage rituel français. Elles sont notamment représentées par les nombreux ex-voto en marbre des églises catholiques, inscrits de remerciements adressés à la Vierge. Elles semblent cependant n'être devenues prédominantes sur le territoire qu’à la fin de la période celtique, aux alentours du ier siècle avant J.-C. Lapparition de ce phénomène est encore mal caractérisée, car il s'avère complexe à reconnaître par l'archéologie. 
Distinction entre «offrande par transformation» et «offrande par destination»

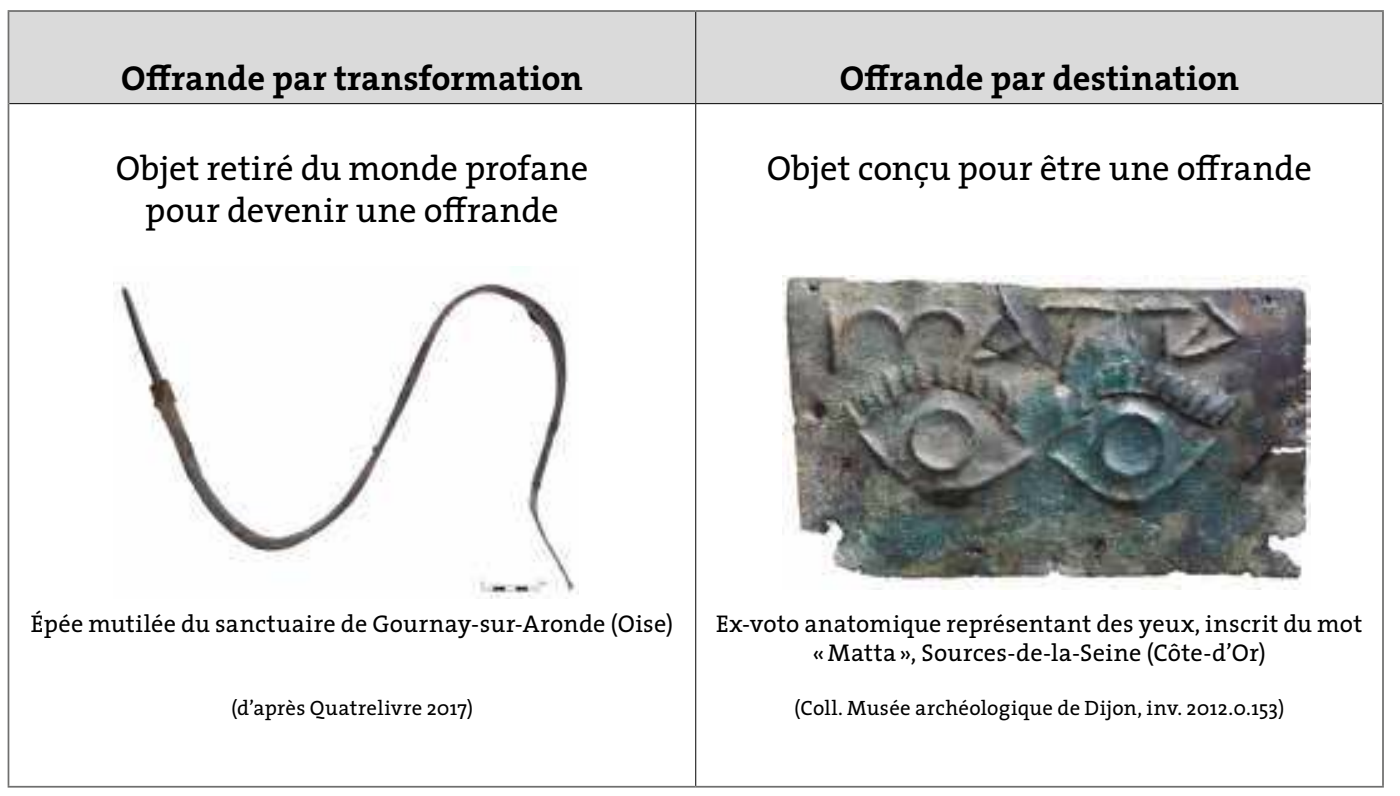

Cet article propose ainsi d'aborder les difficultés particulières de l'étude de l'offrande par destination dans les sociétés celtiques. Après avoir recontextualisé l'apparition de ces nouvelles offrandes au sein de l'évolution des pratiques rituelles au second âge du Fer, les problèmes méthodologiques propres à leur étude par l'archéologie seront exposés, avant de proposer des solutions pour les pallier.

\section{I'apparition de l'offrande par destination}

\section{Un moment clé dans l'évolution des pratiques rituelles celtiques}

Durant la période laténienne, au tournant du $\mathrm{ni}^{\mathrm{e}}$ siècle avant notre ère, une véritable révolution s'opère au sein des pratiques rituelles: les sanctuaires, au sens d'espaces rituels séparés et réservés, apparaissent. Ils témoignent d'une nouvelle organisation des rites, mais aussi d'un changement au sein de leur conception.

Il est possible de distinguer trois phases dans l'évolution des pratiques en cours dans ces sanctuaires à partir des offrandes qui y ont été déposées (Cazanove \& Joly 2011, Bataille 2011, Fauduet 1993, Rey-Vodoz 1991). La première phase, qui s'étend de l'apparition de ces lieux au milieu du II $^{e}$ siècle avant J.-C., est caractérisée par le don de panoplies guerrières et de parures féminines. Il s'agit d'offrandes par transformation: les pratiques pré-dépositionnelles qui leur ont été appliquées confirment bien ce statut. 
Sur les armes, par exemple, il s'agit de dégradations, préférentiellement localisées sur les parties fonctionnelles et blessantes (Bataille 2015 : 151). Au sein du sanctuaire de Gournay-surAronde dans l'Oise, principalement actif à la Tène C et Dl (de 150 à 80 avant J.-C. environ), on a ainsi découpé les tranchants de fers de lances, déformé des umbones - protections centrales de boucliers - par des coups et tordu des épées, au point de les replier sur elles-mêmes (Quatrelivre 2017 : 33, 84, 97). La localisation de ces dégradations a conduit à assimiler ce type de pratiques à un sacrifice de l'objet (Insoll 2011: 151), dans le but de le démilitariser (Bataille 2015: 161). Quelles que soient les intentions qui ont motivé ces actes - retrait d'une arme à un ennemi ou transformation de celle d'un membre du groupe (Bataille et al. 2014: 138) - la perte du caractère fonctionnel des objets permet de les soustraire au monde profane pour les consacrer, et ainsi les transformer en offrande.

La deuxième phase identifiée au sein des pratiques rituelles réalisées dans les sanctuaires celtiques débute au milieu du $\mathrm{II}^{\mathrm{e}}$ siècle avant notre ère, au moment de l'émergence des oppida, c'est-à-dire du développement d'un maillage urbain sur tout le territoire celtique. Elle se caractérise par une véritable diversification des objets déposés: bien que les armes tendent à disparaître, on voit émerger, parmi les offrandes, de la vaisselle, des ustensiles culinaires, potentiels témoins de pratiques de consommation collective, et des objets issus de la sphère artisanale comme de l'outillage (Bataille 2011: 656).

La troisième phase, enfin, est marquée par l'apparition des offrandes par destination, constituées d'objets produits dans le but d'être offerts. En sanctuaire, ils sont donc découverts en position d'offrande, soit primaire, à l'endroit où ils ont été déposés, soit secondaire, lors des cas de nettoyage ou de destruction des sanctuaires. Étant destinées à ne pas appartenir à la sphère profane, ces offrandes n'ont pas besoin d'être sacrifiées et n'impliquent ainsi ni destruction, ni violence (Insoll 2011 : 151, Bataille 1974: 67, Hubert \& Mauss 1899: 88) ${ }^{1}$.

Ce phénomène, attesté dans le monde grec dès le début de l'époque archaïque au viII siècle avant J.-C. (Polignac 1984), ne semble devenir prégnant qu'à partir du Ier $^{e r}$ siècle avant J.-C. dans le monde celtique (Kiernan 2009). Ces nouvelles offrandes sont un des témoignages des profondes transformations apparaissant lors de la conquête. Cette période, qui a pu être qualifiée de «romanisation», correspond à une évolution complexe, hétérogène et inégale des sociétés

Évolution des pratiques rituelles celtiques: état de la question

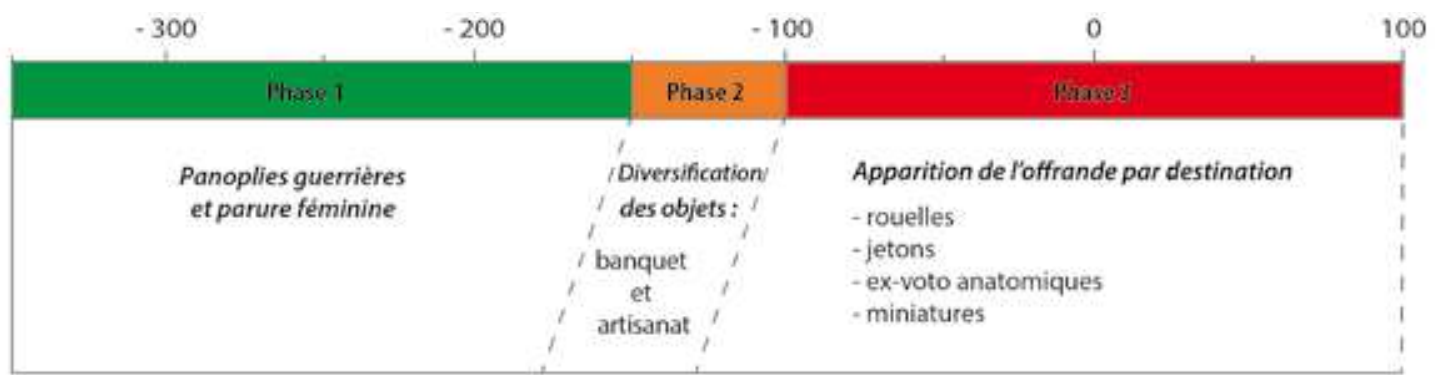




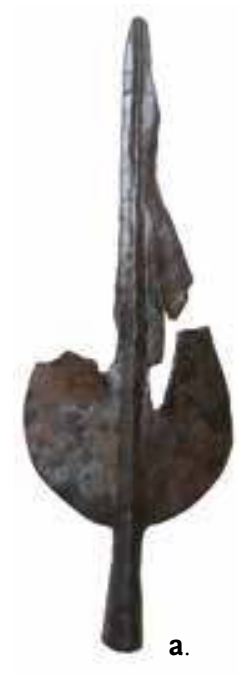

Offrandes par transformation

découvertes au sein du sanctuaire de Gournay-sur-Aronde (Oise)

a. fer de lance;

b. umbo de bouclier:

c. épée;

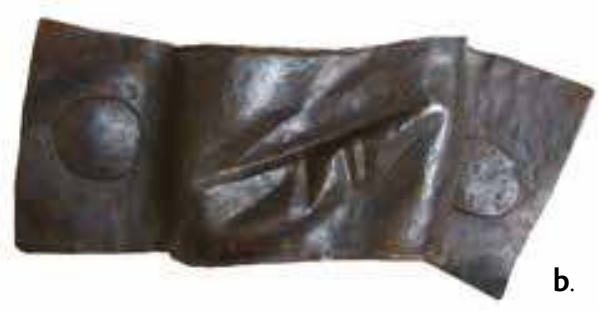

b.

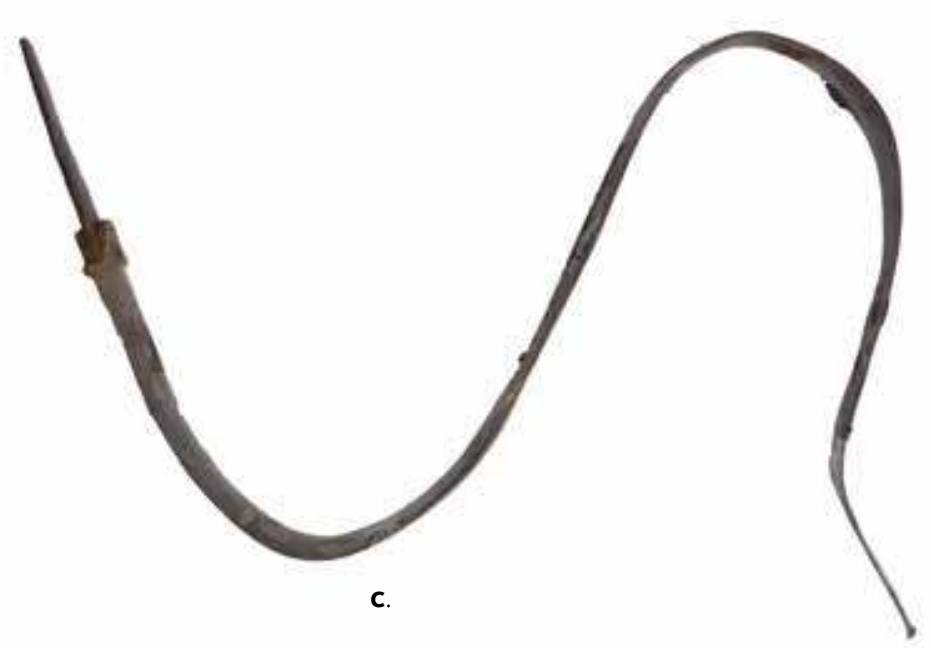

protohistoriques dont la dynamique interne et l'entrée dans la zone d'influence romaine ont entraîné un rapprochement avec le mode de vie italique (Reddé 2011: 9). Les offrandes par destination adoptent cependant des formes différentes dans les sphères méditerranéennes et celtiques. Aux statuettes et ex-voto anatomiques, nombreux dans le monde gréco-romain, sont préférés les armes ou outils miniaturisés, les rouelles et les jetons. Ces offrandes par destination bénéficient de plus de modalités de dépôt particulières : elles sont découvertes par milliers dans les fossés des sanctuaires. Ainsi, plus de 70000 rouelles en or, en argent, en bronze et en plomb ont été retrouvées au sein du sanctuaire de Villeneuve-au-Châtelot dans l'Aube (Piette $\&$ Depeyrot 2008, Bataille 2011 : 658). Elles constituent de véritables séries, composées d'objets de facture et de qualité diverses, reprenant toutefois les mêmes codes de représentation.

L'importance quantitative de ces découvertes d'offrandes semble être un trait caractéristique des sanctuaires de la fin de la période celtique, qui s'estompe au début de la période romaine. En effet, des offrandes par destination y sont toujours présentes, mais en moindre quantité. Cela pourrait correspondre, comme le propose V. Rey-Vodoz, à un changement de conception entre les rites laténiens et la religion romaine.

Il est notamment possible d'aborder la gestion des dons dans le monde romain à travers une loi inscrite sur une pierre du sanctuaire de Jupiter à Furfo, en Italie, en 58 avant J.-C. Cette dernière affirme le droit d'utiliser et de vendre ce qui a été donné. Elle précise même: «Sei quod ad eam aedem donum datum, donatum dedicatum/que erit, utei liceat oeti/venum dare; ubei venum datum erit, id profanum esto.» («Si quelque chose a été donné et dédié à ce temple, qu'il soit permis de l'utiliser, de le vendre. Lorsque cela aura été vendu, qu'il devienne profane».) (ReyVodoz 1991 : 219). Il semble en l'occurrence s'agir davantage d'un transfert, d'un changement de valeur de l'objet offert que d'une véritable perte. Les bénéfices générés par ces ventes sont en effet destinés à mettre en valeur le temple. De ce fait, l'argent ainsi acquis devient consacré (Rey-Vodoz 2006: 236-237). Cette loi ouvre donc la possibilité à la vente, à la refonte et au 


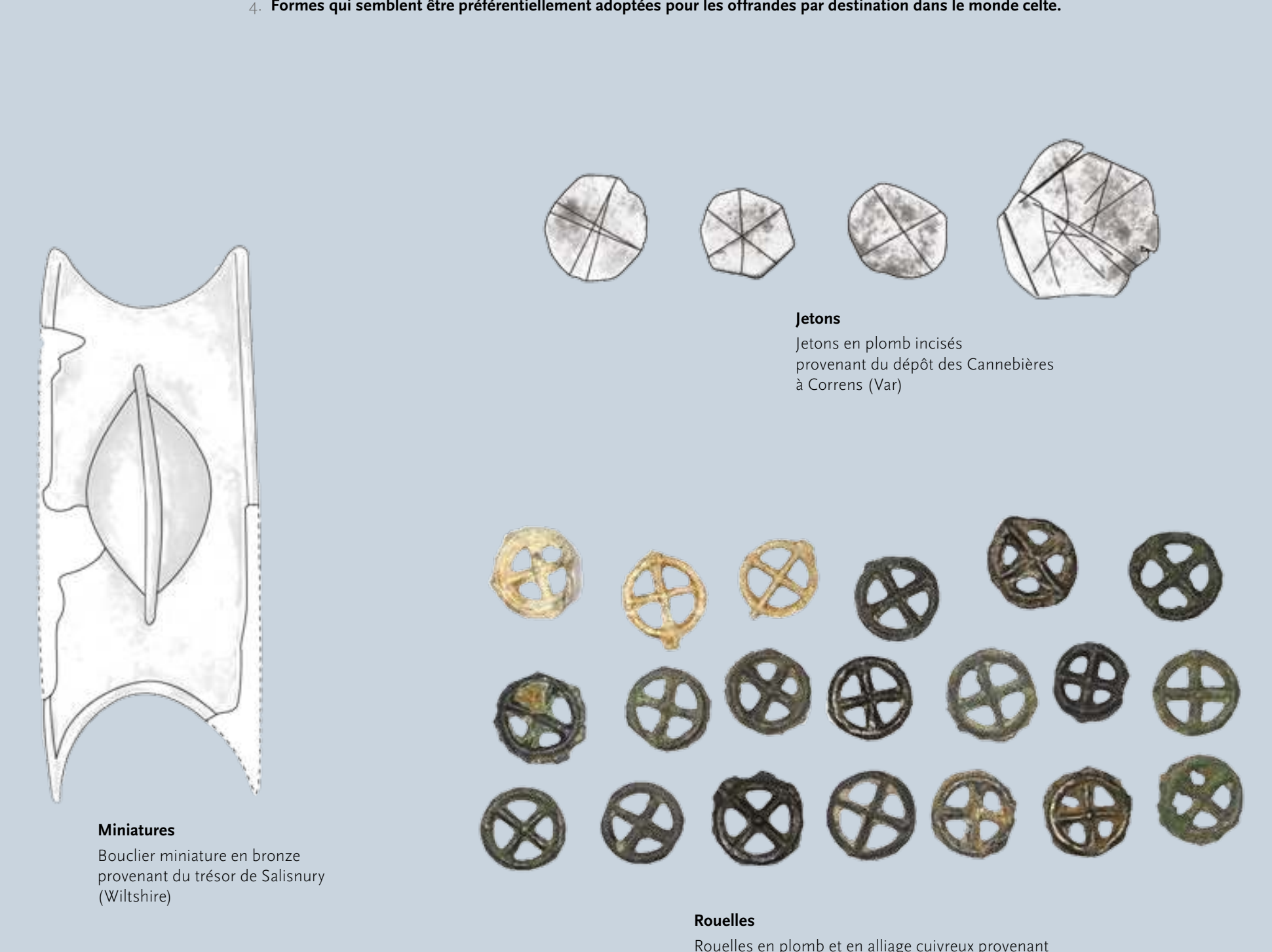


recyclage des offrandes, et donc à la réduction du nombre de ces objets découvert lors de fouilles archéologiques.

Au contraire, selon les écrits romains, notamment César (BG, 6, 17) et Strabon (Géographie: $4,1,13)$, un interdit aurait été promulgué concernant la récupération des offrandes déposées dans les sanctuaires celtiques. Elles semblent y être considérées comme des propriétés inaliénables du sanctuaire (Rey-Vodoz 2006: 237).

Les formes particulières adoptées par ces offrandes pourraient également résulter des essais et des expériences propres aux phases de transitions. Par exemple, le fait de préférer le métal pour ces objets, ou de miniaturiser des armes, comme à Mouzon dans les Ardennes, pourraient correspondre à un attachement à certains traits caractéristiques des pratiques rituelles des phases précédentes, malgré l'adoption d'un nouveau type d'offrande.

Malheureusement, il est encore difficile de bien caractériser l'apparition de l'offrande par destination dans ce monde celtique en mutation, en raison de la difficulté, pour l'archéologie, à identifier ces objets. Il a de plus été montré que certaines offrandes par transformation ont pu bénéficier, à la même période, de modalités de dépôt identiques (cf. le cas d'Acy-Romance, Goussard 2016). Une question vient alors: quels sont les problèmes méthodologiques posés par l'étude de tels objets au sein des sociétés celtiques et dans quelle mesure est-il possible d'y remédier?

\section{Étudier les dons votifs malgré le caractère intangible de l'immatériel}

\section{Le casse-tête archéologique}

L'archéologie est une méthode qui consiste à étudier les sociétés, passées ou présentes, par l'étude de leurs traces matérielles (Demoule et al. 2002: 40). La nature des vestiges étudiés ne permet donc pas un accès direct à la part immatérielle des rites. Si, par l'archéologie, il est possible de restituer les gestes qui ont mené à la présence d'objets dans certaines structures et d'aborder certains traitements pré-dépositionnels ou certains actes de dépôt, la signification de ces gestes ne peut être abordée que sous forme d'hypothèse. Cela complexifie grandement l'étude des dons votifs, qui impliquent de nombreux éléments impalpables.

Si l'on reprend l'exemple des armes volontairement dégradées déposées dans le fossé du sanctuaire de Gournay-sur-Aronde, il est possible d'étudier la mise en œuvre des différentes altérations qu'elles ont subies. Nous pouvons distinguer, par exemple, s'il a fallu réchauffer ces objets pour les tordre et donc mettre en place une forge ou si certaines détériorations ont pu être réalisées à froid. En revanche, il nous est impossible de déterminer avec certitude le sens donné à ces traitements et les hypothèses restent multiples, qu'il s'agisse de la valeur que l'on accorde à ces dégradations, ou de l'identité des propriétaires initiaux de ces armes (membre du groupe ou ennemi). 
Pour pallier ce problème, il convient de conjuguer l'approche archéologique avec une approche historique et de se référer aux textes, notamment contemporains, décrivant une partie de ces rites. Toutefois, les sociétés celtiques de l'âge du Fer n'ont malheureusement laissé que de rares traces épigraphiques, dans des alphabets grecs ou latins, et aucun écrit endogène sur les offrandes et leur signification. Il est ainsi impossible de se référer, comme pour l'étude des cultes grecs ou romains, à l'inventaire d'un temple mentionnant des offrandes, à l'exemple de Délos (Prêtre 2014), ou de connaître en détail toutes les étapes d'un rite, comme c'est le cas avec Les Actes des Frères Arvales, pour la période romaine (Scheid 2005).

Pour la période celtique, ces difficultés font de l'offrande le vecteur d'accès privilégié aux rites de dons votifs. Elle constitue en effet l'expression la plus concrète et matérielle de ce don à une entité supposée agissante (Testart 2012: 435). En archéologie celtique, l'étude des rites de dons en sanctuaire se base ainsi sur l'examen des objets offerts et de leur contexte: sur leur analyse intrinsèque, mais aussi sur celle des structures qui les accueillent, de la manière dont ils y ont été déposés et des autres éléments qui les accompagnaient.
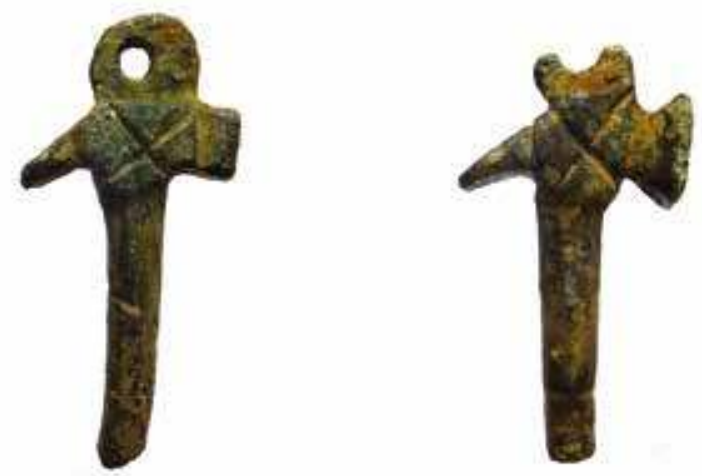

À ces difficultés, propres à l'étude de toute offrande dans une société passée anépigraphe, s'ajoutent celles spécifiques à l'étude des offrandes par destination. Le propre de tels objets est de n'avoir d'autre utilité que d'être offerts et donc de n'avoir aucune fonction technique. Au contraire d'un couteau muni d'une lame comportant un tranchant qui permet de couper, une offrande par destination est dépourvue de partie active.

La reconnaissance de cette absence de partie active et donc de fonction technique peut parfois s'avérer complexe (Goussard 2015: 32). Surtout, comme l'a souligné A. Testart, quand on ne sait pas comment un objet est censé être utilisé (Testart 2012: 327). Pour illustrer ce propos, il est possible d'évoquer le cas d'une série de petits « pendentifs» en bronze en forme de marteau, retrouvés dans
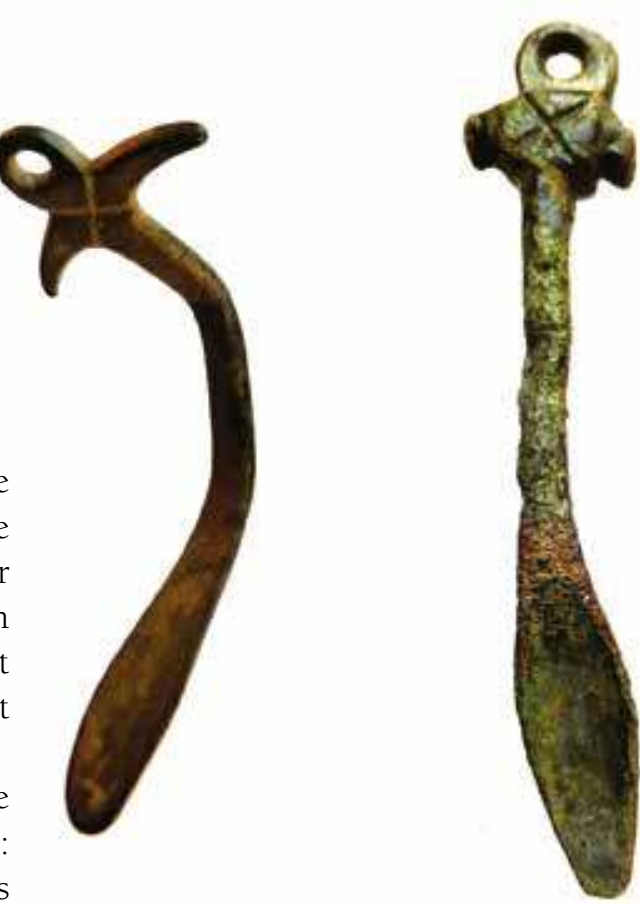

5. Marteaux miniatures ou cure-pipes? le Nord de la France et dans le Hainaut belge. La découverte d'un de ces exemplaires dans la terre arable, au-dessus du sanctuaire de 
Kruishoutem-Kapellekouter en Belgique, avait conduit à assimiler ces objets à du matériel votif romain et plus particulièrement à des représentations miniatures. Ils sont en effet de petites dimensions, adoptent une forme de marteau et ne semblent pas présenter de partie active. Cependant, l'impossibilité de relier avec certitude cette découverte à la période romaine du site et l'absence de comparatif exact parmi les offrandes de cette période ont conduit J. Dufrasnes à chercher des rapprochements avec des objets de périodes plus récentes (Dufrasnes 2004, 2005; Kiernan 2009: 186-187). Au bout de ses recherches, il a montré qu'il était probable qu'il s'agisse de cure-pipes de l'époque moderne. La difficulté à identifier ces objets tenait au fait que la partie inférieure, servant à nettoyer le fourneau, était cassée).

Ainsi, la compréhension de l'apparition et du développement de l'offrande par destination chez les Celtes connaît plusieurs limites. L'étude de tels objets est en effet conditionnée par les problèmes méthodologiques inhérents à l'analyse des rites d'offrandes dans une société anépigraphe, ainsi qu'à ceux liés à l'absence de fonction qui les caractérise. Face à ces difficultés et pour pouvoir appréhender ces rites, il convient donc tout d'abord de mettre en place une méthode et des critères pour reconnaître ces offrandes par destination.

\section{Traces matérielles des désirs des derniers gaulois}

\section{Reconnaître une offrande par destination chez les Celtes}

La première étape consiste à écarter les objets qui ont une fonction technique, notamment parmi les objets premièrement interprétés comme des offrandes par destination. Il est possible de commencer par une étude neutre de la forme des objets, suivie de la mise en place d'une typo-morphologie, c'est-à-dire d'un classement des exemplaires étudiés selon leur forme. Cette approche permet ensuite d'établir une typologie fonctionnelle et donc une classification de ces objets selon leur utilisation. Elle facilite également la comparaison avec les typologies de référence et rend ainsi possible l'exclusion des objets qui ont une partie active et qui, par définition, ne sont pas des offrandes par destination.

Cette démarche a notamment été appliquée sur une série d'objets premièrement interprétés comme des lances miniatures découvertes à Acy-Romance, dans les Ardennes (Goussard 2016). Il s'agit de près de 700 petites pointes en fer munies d'une douille de fixation, rejetées dans un puits au $\mathrm{I}^{\mathrm{er}}$ siècle avant J.-C., au moment de l'abandon de cette agglomération protohistorique. Elles présentent un aspect assez grossier, résultat d'une fabrication rapide et peuvent paraître, au premier abord, comme non fonctionnelles. La classification de ces objets selon une typologie sans a priori interprétatif, basée sur des critères morphologiques et métriques, suivie de la comparaison avec des typologies d'armes de taille, d'estoc et de jet, ont permis d'y reconnaître des armes réelles dans 91,6 \% des cas. La majorité des identifications a été effectuée à la suite de la comparaison avec les corpus de pointes de flèches de contextes militaires de la Guerre des Gaules (Goussard et al., à paraître). 
Cette approche comporte cependant des limites: l'évolution des connaissances et la découverte de nouveaux types d'objets peuvent remettre en cause les interprétations réalisées. Par exemple, les progrès effectués en matière de reconnaissance et d'identification des matériaux périssables en archéologie ont grandement modifié la perception que nous avons de l'utilisation de certains vases. Il est possible d'évoquer le cas des hydries, des vases grecs dont le nom dérive de to hudôr, servant à désigner l'eau en grec ancien. Leur fonction première était ainsi de contenir et de puiser l'eau. Des analyses menées sur six hydries découvertes dans une tombe à proximité du temple d'Athéna à Paestum ont cependant révélé des traces de miel à l'intérieur de ces contenants. Cette découverte a amené à diversifier les interprétations quant à l'utilisation de ces vases, et notamment à envisager une dimension religieuse (Sestieri 1956, cité par Sowder 2009: 385). Ce type d'analyse pourra peut-être nous prouver que certains vases de très petites dimensions, interprétés comme non fonctionnels, contenaient en réalité du parfum ou des denrées alimentaires.

Au-delà de cette démarche visant à exclure du champ d'étude les objets dont la fonction technique a été identifiée, il est possible de repérer des indices de

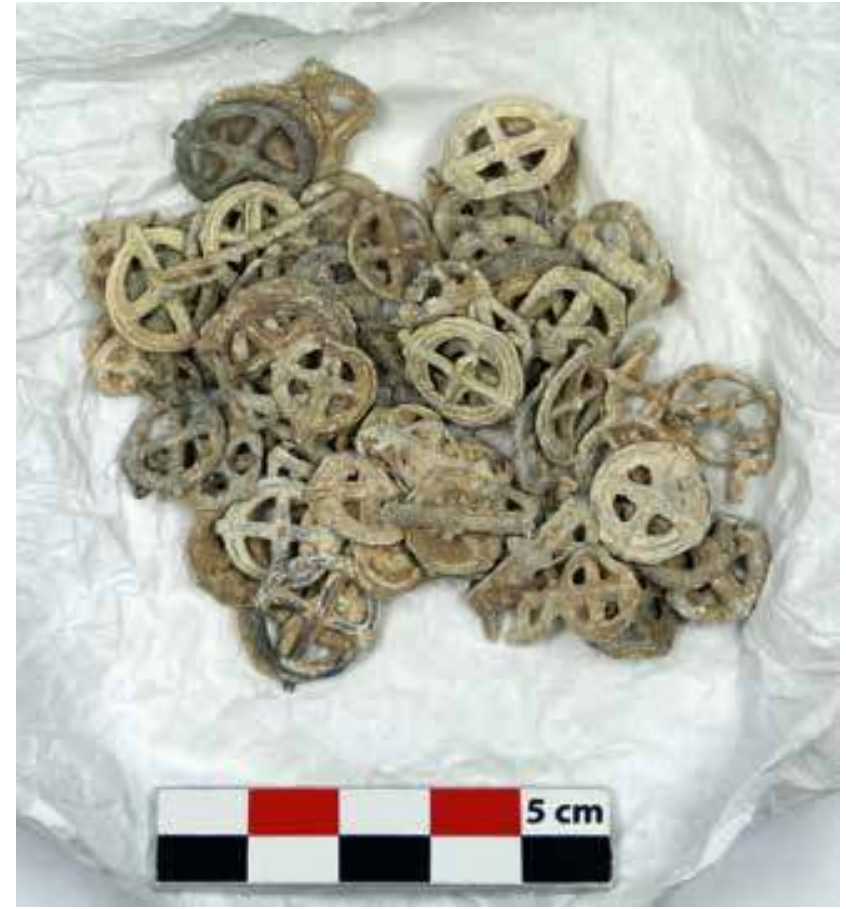

6. Rouelles brutes de coulées provenant du sanctuaire de Nanteuil-sur-Aisne (Ardennes).

l'«a-fonctionnalité » voulue pour ces objets. La présence dans certains sanctuaires de séries d'offrandes brutes de coulées auxquelles aucune finition n'a été apportée en est un exemple. C'est le cas notamment des rouelles en plomb retrouvées par centaines dans le sanctuaire de la Villeneuve-au-Châtelot dans l'Aube (Piette \& Depeyrot 2008), de Nanteuil-sur-Aisne, dans les Ardennes (Lambot 1989), ou encore du Haut du Bois de Pique à Moyencourt, dans la Somme (Desforges 2010). Certaines d'entre elles sont découvertes «en chapelet ». En effet, afin d'en faciliter et d'en accélérer la production, un même moule est utilisé pour produire plusieurs de ces objets. Pour que le métal en fusion puisse remplir en un seul temps tout l'espace libre du moule, des petits sillons sont creusés entre chaque négatif de rouelle. Au moment du démoulage, c'est donc « un chapelet » de rouelles qui en est extrait. Il est intéressant de noter qu'un phénomène similaire est attesté pour les 400 anneaux de la favissa - un espace réservé pour la mise au rebut, au sein du sanctuaire, d'objets consacrés - découverts à Baron-sur-Odon dans le Calvados (Bertin 1977: 82-83). Certains sont associés par deux ou trois; tous ceux qui ont été individualisés comportent les traces d'une cassure réalisée au burin, qui n’a pas été masquée. Ce type d'irrégularité est habituellement effacé par abrasion, notamment lors de la réalisation d'objet de parure. 
Il faut également noter que les dépôts massifs de ces séries d'objets non finis coïncident avec une inflation des découvertes monétaires en sanctuaire. La mutation des pratiques rituelles à la fin de la période celtique, et particulièrement l'apparition de ces nouvelles offrandes, a pu entraîner le développement d'une nouvelle économie au cœur de ces lieux (Martin 2015: 166169). La nécessité d'acquérir ces objets a pu participer à la construction d'un marché des rites et des désirs.

L'offrande par destination semble occuper, au sein de l'évolution des pratiques rituelles celtiques, un moment charnière, témoin de profondes transformations. Le fait que la part immatérielle des rites ne soit pas accessible archéologiquement et l'absence de textes endogènes pour pallier ce problème complexifient l'étude de ces offrandes. En outre, pour identifier une offrande par destination, il faut pouvoir prouver son absence de fonction, démarche qui est souvent tributaire de l'évolution de nos connaissances.

Pour appréhender ce phénomène et ne pas en fausser la vision, il convient de veiller à écarter les objets qui ont une fonction, et donc les offrandes par transformation. Pour cela, il faut réaliser, comme précédemment pour l'analyse des offrandes par destination, une étude neutre des objets, sans a priori interprétatif, et mettre en place une typo-morphologie permettant, dans un second temps, d'aboutir à une typologie fonctionnelle. Cette démarche se doit d'être complétée par la recherche d'indices d'a-fonctionnalité, comme le choix de laisser certaines offrandes sans finition ou même brutes de coulée. Leur absence d'utilisation en dehors du don ne rend en effet pas nécessaires ces dernières étapes de mise en forme.

Les problèmes méthodologiques mis ici en évidence montrent ainsi la nécessité, pour aborder l'offrande par destination chez les Celtes, d'analyser l'expression concrète de ces dons. En effet, la rareté des sources permettant d'étudier de telles pratiques amène à questionner de nouveau l'objet lui-même. Cela conduit en particulier à s'interroger sur l'impact de l'immatériel sur le matériel, sur les conséquences du statut d'offrande par destination dans la matière même d'un objet.

De telles transformations ne sont pas anodines: elles concernent des productions situées à la frontière entre la sphère profane et la sphère sacrée, qui sont également de véritables liens entre le dédicant, l'entité à laquelle il offre, mais aussi le groupe social auquel il appartient (Polignac 2009). Elles peuvent ainsi autant témoigner d'intentions individuelles, à l'image des velléités ostentatoires de Bohémond de Tarente, que de profonds changements au centre de l'organisation et de la conception de la sphère sacrée.

\section{Notes}

1. Soulignons, comme l'a fait R. Caillois, que toutes ces normes peuvent être détournées, de façon anec-

dotiques (Caillois 1950 : 24). Dans le sanctuaire des Flaviers à Mouzon (Ardennes), par exemple, sur 
les 419 boucliers miniatures offerts à la fin de la période celtique, 12 (ou 2,8\%) ont été volontairement endommagés. De même, sur les 451 épées miniatures offertes, 14 (ou 3,2\%) présentent des traces de dégradations volontaires (sous forme de pliures, d'entailles ou de sectionnement) (Caumont 2011).

\section{I'auteure}

Élisabeth Goussard prépare actuellement une thèse à l'École Pratique des Hautes Études sous la direction de Stéphane Verger, dont le sujet porte sur l'apparition de l'offrande par destination en Gaule celtique, entre le inle s. av. J.-C. et le $\mathrm{I}^{\mathrm{er}}$ siècle de notre ère.

\section{Iconographie}

Image d'ouverture. Boucliers miniatures découverts au sein du sanctuaire des Flaviers à Mouzon. Hauteur comprise entre 3,5 et $17,3 \mathrm{~cm}$. (C) E. Goussard.

1. C E. Goussard d'après G. Bataille, I. Fauduet \& V. Rey-Vodoz.

2. (C) E. Goussard, d'après Cazanove \& Joly 2011, et Bataille 2011.

\section{Références}

Bataille, Geo. 1974 Théorie de la religion. Texte établi et présenté par Thadée Klossowski. Paris: Gallimard.

Bataille, Gér. 2015 «Approches des pratiques rituelles. Proposition de restitution à partir des dépôts terrestres non funéraires à composante métallique» in J. Kaurin et al. dir. Décrire, analyser, interpréter les pratiques de dépôt de l'âge du Fer. Actes de la table ronde de Bibracte, (Glux-en-Glenne, 2012). Glux-en-Glenne: Bibracte: 145-164.

— 2011 «Principes d'évolution des ensembles de mobiliers métalliques issus de sanctuaire, dans le quart est de la Gaule, entre les $\mathrm{III}^{\mathrm{e}}$ S. av. - $\mathrm{II}^{\mathrm{e}}$ S. apr. J.-C.» in M. Reddé et al. dir. Aspects de la Romanisation dans l'Est de la Gaule. Glux-en-Glenne: Bibracte: 655-662.

Bataille, Gér. et al. 2014 «Une archéologie de la guerre au second âge du Fer (fin du ive siècle av. - début du $\mathrm{i}^{\mathrm{er}}$ siècle apr. J.-C.) » in O. Buchsenschutz et al. dir. Archéologie de la violence et de la guerre dans les sociétés pré et protohistoriques. Paris: CTHS - édition électronique: 129-141.

Bertin, D. 1977 «Le sanctuaire celto-romain du Mesnil de Baron-sur-Odon (Calvados)», Gallia 35(1).

BHL 1898-1901 Bibliotheca hagiographica latina antiquae et medice atatis (BHL). Société des Bollandistes: Bruxelles.
3. (C) D'après Quatrelivre 2017, fig. 20 : 33, fig. 84: 111, fig. $97: 122$.

4. DAO : Élisabeth Goussard d'après Kiernan 2009 (C) E. Goussard.

5. C Clichés J. Dufrasnes.

6. (C) E. Goussard.
Caillois, R. 1950 L'homme et le sacré. Paris: Gallimard. Caumont, O. 2011 Dépôts votifs d'armes et d'équipements militaires dans le sanctuaire gaulois et gallo-romain des Flaviers à Mouzon (Ardennes). Montagnac: Monique Mergoil.

Cazanove, O. \& M. Joly 2011 « Les lieux de culte du nord-est de la Gaule à l'époque de la romanisation. Sanctuaires ruraux, sanctuaires d'agglomération, rites et offrandes» in M. Reddé et al. dir. Aspects de la romanisation dans l'Est de la Gaule. Glux-en-Glenne: Bibracte: 663-669.

Cheirézy, C. 1995 «Hagiographie et société: l'exemple de saint Léonard de Noblat », Annales du Midi. Revue archéologique, historique et philologique de la France méridionale. Tome 107 (212): 417-435.

Demoule, J.-P. et al. 2002 Guide des méthodes de l'archéologie. Paris: La Découverte.

Desforges, J. 2010 «Le Haut du Bois de Pique» à Moyencourt (Somme). Rapport de diagnostic (INRAP, GB19911401). Amiens: DRAC-SRA Hauts-de-France.

Dufrasnes, J. 2004 «Pendentifs en forme de marteau », Instrumentum 20 (16).

- 2005 «Énigmatiques pendentifs en forme de marteau découverts dans le nord de la France et dans le Hainaut 
Belge », Société Tournaisienne de Géologie, Préhistoire et Archéologie 9: 205-232.

Fauduet, I. 1993 Les temples de tradition celtique en Gaule romaine. Paris: Errance.

Goussard, E. 2016 Étude du mobilier métallique de la structure 02 7109, dite «le puits aux lances miniatures», d'Acy-Romance. Paris: École Pratique des Hautes Études, mémoire de master 2.

— 2015 « La miniature et l'offrande, réflexions autour des armes miniatures celtiques», Histoire de l'art $77: 31-42$

Goussard et al., à paraître «Le phénomène des dépôts de miniatures d'armes dans les sanctuaires celtes. Réflexions à partir de l'exemple des Rèmes » in P. Barral et al. dir. Sanctuaires de l'âge du Fer, Actes du 41e colloque de l'AFEAF (Dole, 2017).

Hubert, H. \& M. Mauss 1899 Essai sur la nature et la fonction du sacrifice. Paris: Félix Alcan.

Insoll, T. dir. 2011 The Oxford Handbook of the Archaeology of Ritual and Religion. Oxford: Oxford University Press.

Kiernan, P. 2009 Miniature Votive Offerings in the Roman North-West. Ruhpolding: Verlag Franz Philipp Rutzen.

Lambot, B. 1989 «Le sanctuaire gaulois et gallo-romain de Nanteuil-sur-Aisne, lieu-dit Nepellier (Ardennes)», Bulletin de la Société archéologique champenoise 82(4): 33-44.

Martin, S. 2015 Du statère au sesterce. Monnaie et romanisation dans la Gaule du Nord et de l'Est (III' s. a.C.- I I p.C). Bordeaux: Ausonius Éditions.

Morel, J.-P. 1992 «Ex-voto par transformation, ex-voto par destination (à propos du dépôt votif de Fondo Ruozzo à Teano)», Mélanges Pierre Lévêque. Tome 6: Religion. Besançon: université de Franche-Comté: 221-232. (Annales littéraires de l’université de Besançon 463).

Piette, J., \& G. Depeyrot 2008 Les monnaies et les rouelles du sanctuaire de La Villeneuve-au-Châtelot (Aube) (II ${ }^{e}$ siècle av. J.-C. - ve siècle apr. J.-C.). Wetteren: Moneta.
Polignac, F. 1984 La naissance de la cité grecque. Cultes, espace et société, $\mathrm{VIII}^{e}-\mathrm{VII}^{e}$ siècles avant J.-C. Paris: La Découverte.

- 2009 «Quelques réflexions sur les échanges symboliques autour de l'offrande» in C. Prêtre dir. Le donateur, l'offrande et la déesse. Systèmes votifs des sanctuaires de déesses dans le monde grec. Liège: Presses universitaires de Liège: 29-37.

Prêtre, C. 2014 «L'offrande dans les inventaires de Délos: objet rituel ou sacré? », Revue de l'histoire des religions 231(4): 539-557.

Quatrelivre, C. 2017 Le sanctuaire de Gournay. Histoire de la découverte, état de la documentation actuelle, nouvelles analyses du mobilier métallique. Paris: École normale supérieure, mémoire de master 2.

Reddé, M. et al. dir. 2011 Aspects de la Romanisation dans l'Est de la Gaule. Glux-en-Glenne: Bibracte.

Rey-Vodoz, V. 1991 «Les offrandes dans les sanctuaires gallo-romains » in J.-L. Brunaux dir. Les sanctuaires celtiques et leurs rapports avec le monde méditerranéen. Actes du colloque de Saint-Riquier (novembre 1990). Paris: Errance, 215-220.

- 2006 «Offrandes et rituels votifs dans les sanctuaires de Gaule romaine » in M. Dondin-Payre \& M.-T. Raepsaet-Charlier dir. Sanctuaires, pratiques cultuelles et territoires civiques dans l'Occident romain. Bruxelles: Le Livre Timperman, 219-237.

Scheid, J. 2005 Quand faire, c'est croire. Les rites sacrificiels des Romains. Paris: Aubier.

Sowder, A. 2009 Greek Bronze Hydriai. Atlanta: Emory University.

Testart A. dir. 2012 Les armes dans les eaux, questions d'inter -prétation en archéologie. Paris: Errance.

\section{Pour citer l'article}

Goussard, E. 2018 «Produire pour offrir. L'offrande par destination chez les Celtes», Techniques\&Culture 70 « Matérialiser les désirs. Techniques votives», p. 200-213. 
